\title{
Escritura y reescritura extraescolares: aspectos lingüísticos y cognitivos
}

Jacques David ${ }^{2}$

\section{Introducción}

$\mathrm{E}$ ste estudio se apoya en los datos de una investigación tendiente a describir el proceso de escritura de alumnos del ciclo tercero de escuela primaria' (8-11 años). Con este propósito, recogimos un corpus de más de un centenar de textos producidos al margen de actividades escolares: cuentos, relatos de experiencias personales, diarios íntimos, poemas, adivinanzas, chistes, enunciados de problemas matemáticos e incluso una obra de teatro. Estos textos, recogidos con la autorización de los niños, nos sirvieron de base para el estudio de diferentes procedimientos de relectura y reescritura comprometidos en este proceso. Presentamos aquí solamente una parte de un análisis más amplio de las versiones y borradores recopilados, así como del trabajo metalingüístico presente en estos textos. Centraremos nuestro propósito en algunos problemas de ortografía y de composición. Nuestros trabajos se sitúan en el campo de las investigaciones lingüísticas y psicolingüisticas, por lo tanto, pretenden contribuir a la reflexión emprendida en estos últimos años sobre una didáctica de la escritura que tenga en cuenta estas producciones extraescolares originales con el fin de favorecer su emergencia e incluso su práctica regular.

\section{Marco de referencia}

\subsection{De las investigaciones centradas en el proceso de escritura...}

En los últimos veinte años han aumentado considerablemente los trabajos que se interesan en los procesos de escritura, distinguiendo aquello que atañe al estudio de los productos -dicho de otra manera, las huellas o resultados de la escritura, constituidos por textos más o menos terminados- del análisis de los mecanismos cognitivos que los generan. Tradicionalmente, las investigaciones que intentan describir los procesos de escritura pertenecen en primer lugar a los paradigmas de la psicología cognitiva (Fayol, 1997, y Piolat y Pélissier, 1998) y tienen en cuenta algunas veces las implicaciones neuropsicológicas (Zesiger, 1995). Estas investigaciones tienen por objeto, no los resultados de la producción escrita, sino los diferentes procedimientos movilizados por un sujeto aprendiz o experto. Para ello, estos trabajos han tenido que modificar sus metodologías. En efecto, hasta los años 70

1 Traducido por Raquel Pinilla Vásquez y Blanca Lilia Bojacá Bojacá, grupo de investigación Lenguaje, Identidad y Cultura, Universidad Distrital Francisco José de Caldas, Bogotá, 2008.

2 IUfm de Versailles CNRs “Leaple”, umr 8606 y París 5. Tomado de revista Repères, (2001) núm. 23, número dirigido por Ives Reuter y Marie-Claude Penloup, pp.33-54, INRP. 


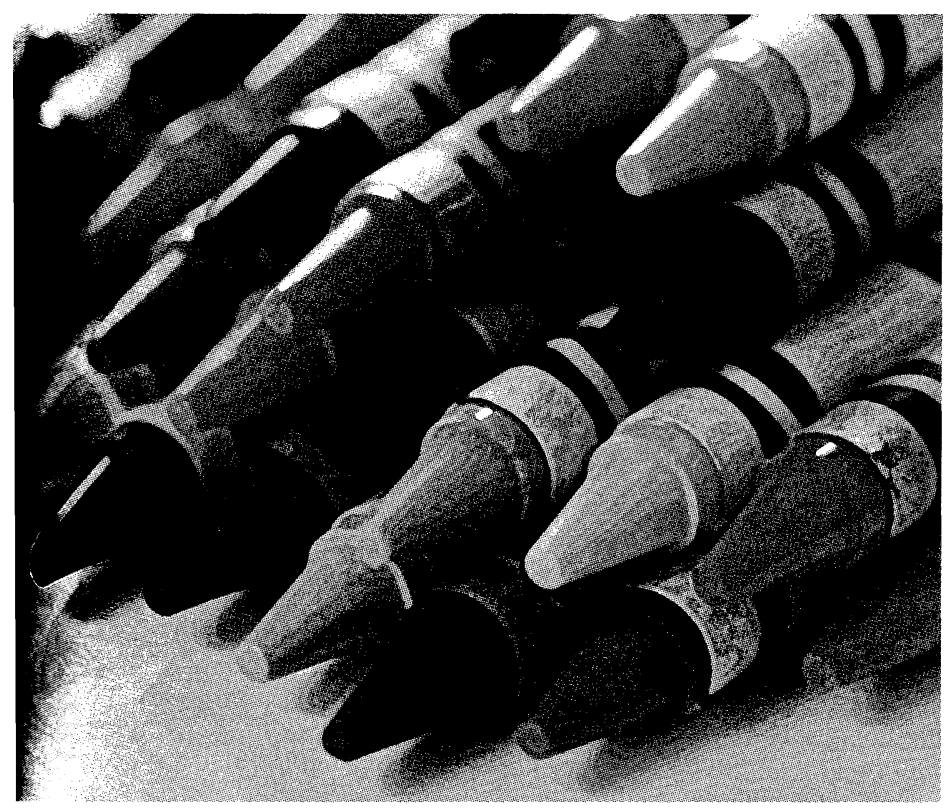

las investigaciones en psicología, inspiradas aún por el modelo constructivista, inferían la existencia de competencias de escritura a partir del análisis de textos terminados (Simon, 1973). E1 objetivo consistía entonces en describir los procedimientos psicolingüísticos más cercanos a las exigencias lingüísticas requeridas en el lenguaje escrito: la construcción de frases, el manejo de pronombres y el funcionamiento de los verbos..., todo esto relativizando la norma escrita con el fin de privilegiar el estudio del proceso de escritura y las diferentes fases de su adquisición.

Hoy día, las metodologías experimentales pretenden aproximarse más directamente a los actos de escritura sin pasar por la interpretación de los textos producidos. Estos procesos son ilustrados por Piolat y Pélissier (1998), quienes constatan que desde comienzos de los años 80 la perspectiva cognitiva intenta analizar más de cerca los procesos de redacción (método on-line), allí donde antes no se interesaba sino en los resultados, es decir, en los productos obtenidos de la actividad (método off-line). Sin embargo, varias consecuencias resultan de esta evolución. En efecto, si los métodos on-line relacionados con "la colección de datos sincrónicos en la composición de textos" volvieron caducos los métodos de interpretación de los textos escritos, esos métodos descuidaron el análisis de "los diferentes niveles lingüísticos de los textos redactados" (229). Teniendo en cuenta estas afir- maciones mostraremos en las páginas que siguen que el análisis de la actividad escritora, que comprenda una observación inmediata, no puede aislarse del estudio de las exigencias lingüísticas que están en juego en ese proceso.

Esta misma perspectiva es compartida por otros, especialmente por Fayol (1997), quien no duda en declarar que de ahora en adelante es necesario completar estos métodos en tiempo real con estudios empíricos que no descuiden los factores lingüísticos y su tratamiento en una tarea de producción escrita. Esta orientación en la investigación está apenas en sus comienzos; en efecto, se requiere multiplicar los trabajos en este campo para medir con mayor precisión el impacto de todas las exigencias lingüísticas en la adquisición y desarrollo de una competencia tan compleja como la escritura.

Como se ha visto, no tiene mucho sentido oponer el estudio de la producción escrita -en el experto como en los aprendices-al del componente lingüístico, se trate del dominio de la composición o de la organización globảl de un texto. Es el caso del estudio de la adquisición de la ortografía, de la puntuación, de la cohesión tempo-verbal, que revela fenómenos discursivos integrados cada vez más en las descripciones y modelaciones lingüísticas. Si queremos superar nuestro desconocimiento actual de la génesis de operaciones lingüísticas tan complejas, debemos a la vez plantear modelos explicativos suficientemente coherentes y describir procedimientos bastante precisos. Para ello, el estudio de los textos de los alumnos, producidos en medio escolar o extraescolar, va de la mano con el estudio del proceso cognitivo.

\section{2. ... a las investigaciones centradas en los textos de los alumnos}

La mayor parte de los trabajos que se proponen describir textos de niños (o de adolescentes) tienen generalmente una orientación didáctica. También han sido el fruto de un trabajo que reposa sobre la constitución de un corpus más o menos importante, en sincronía (más frecuente) o en diacronía (más rara). La escuela o el aula es el lugar privilegiado para recoger estos textos, sencillamente porque es el marco en el cual los jóvenes escritores pueden producir bastante. En ese 
sentido, hablaremos de textos de alumnos y no en general de textos de niños, incluso si el estudio propuesto aquí describe textos de escolares producidos en espacios exteriores a la escuela (ver igualmente, a este propósito, Escritos de colegiales, Penloup et al., 1999).

El estudio de los escritos de los alumnos, ya sean escolares o extraescolares, dista mucho de ser un trabajo acabado; sin duda porque este estudio comprende, además de los textos recogidos; otras informaciones: los diferentes soportes (cuadernos, libretas, carpetas, hojas sueltas o argolladas), las ilustraciones (fotografías, viñetas, objetos pegados) y las diversas versiones de reescritura correspondientes, pero también, más recientemente, las observaciones de investigadores sobre los comportamientos de los alumnos y, aún más, los comentarios producidos durante o al final de la producción (Jaffré y $\mathrm{Du}-$ card, 1996; Bousquet et al. 1999, y nuestros propios trabajos en las páginas que siguen). ${ }^{3}$

Sin embargo, las primeras investigaciones de gran magnitud ligadas a este paradigma encuentran su origen en las de Fabre (1990). Su corpus de más de 300 borradores producidos en las diferentes aulas de la escuela elemental (más de 6000 enmendaduras o tachones) es suficiente para el análisis de las diferentes operaciones de reescritura realizadas por los jóvenes escritores. Más allá de los resultados cuantitativos, Fabre validó hipótesis lingüísticas formuladas anteriormente en un marco teórico hasta ese momento poco explorado, el de la crítica genética (Hay, 1979, y Grésillon, 1994). Esta autora también puso en evidencia que los tacho-

\footnotetext{
3 A estos trabajos habría que agregar aquellos que se apoyan en corpus de textos espontáneos o inducidos por copia, dictado, advertencia, y que pretenden describir precisamente la génesis o psicogénesis del lenguaje escrito en los niños pequeños (especialmente los trabajos de Ferreiro y Teberosky 1979, 1982, y Ferreiro 1988, 2000, retomados por otros, especialmente en Francia: Fijalkow (dir.) 1990; Besse, 1995; Jaffré, 1992, y, para una síntesis, David, 2001).
}

nes de los alumnos dan cuenta de la adquisición y la puesta en marcha de operaciones metalingüísticas particulares, construidas en una relación con la lengua pero también en una relación con el texto en gestación, en los dos planos del contenido y de la organización lingüística, y en fin en una relación con el otro, el lector imaginado o real.

Las descripciones obtenidas de estos trabajos fundadores muestran que la producción textual de un alumno no puede reducirse a un solo componente. De hecho, todo escrito, escolar o no, en borrador o terminado, secreto o editado, manifiesta una complejidad fundamentalmente metalingüística. El estudio de enmendaduras y tachones muestra, en efecto, que estos escritos ponen de relieve un modo particular de expresión, un modo donde la actividad reflexiva es primordial, un modo que combina necesariamente contingencias enunciativas y exigencias lingüísticas. Esta aproximación genética ha inspirado otras investigaciones, a veces de la misma amplitud, sobre géneros particulares (Bucheton, 1995; Tauveron, 1995; Bonnet, Corblin y Elalouf, 1998; Boré, 1998; Fabre (dir.), 2000, y Penloup, 2000) o producidos en contextos de aprendizajes particulares y con orientaciones más o menos didácticas (Barré de Miniac, Cros y Ruiz, 1993; Plane, 1994; David y Plane (edits.), 1996; Plane y Turco (dirs.), 1996, y Reuter, 1996). Estas diferentes investigaciones proponen miradas que oscilan con frecuencia entre la neutralidad descriptiva -lingüística, socio o etnolingüística- y la evaluación de dispositivos de aprendizaje. Pero más allá de las variaciones metodológicas observadas se distinguen en estos trabajos las focalizaciones lingüísticas. Algunas investigaciones se centran definitivamente en el análisis de los componentes morfosintácticos y lexicales de las producciones escritas, otras se apartan de este nivel para acercarse solamente a los aspectos enunciativos y textuales. Son más raras las que se solidarizan con los diferentes componentes. Las nuevas investigaciones 
deben abrirse entonces a esta necesaria complementariedad de los procesos de composición que, desde nuestro punto de vista, es inherente a todo trabajo de escritura e inseparable desde el punto de vista de la adquisición.

Más específicamente aún, sólo algunas investigaciones se han interesado en las prácticas de escritura extraescolares de los jóvenes (Bonnet y Gardes-Tamine, 1990; Blanc, 1996; Penloup (edit.), 1994, y Penloup, 1999), y ninguna -al menos en lo que conocemos- ha descrito con precisión las composiciones de los alumnos de escuela primaria. Las investigaciones realizadas con jóvenes muestran que ellos escriben en proporciones importantes y están abiertos a una diversidad textual relativamente extensa. Este descubrimiento -en el sentido de que se trata con frecuencia de prácticas insospechadas- muestra que los niños y los adolescentes han asumido la escritura más allá de lo esperado, en relación con las prácticas y formas usuales en la escuela. Se inscriben así en una relación con la escritura que es ante todo una relación con el otro y/o con ellos mismos. La expresión de un pensamiento, y también de emociones, de sentimientos, de pequeñas y grandes “ historias", alimenta en abundancia sus escritos personales. Falta por describir con precisión los procesos de escritura en el momento de la producción, y tal vez proponerse tomarlos en cuenta en una didáctica de la escritura ampliada a otros universos distintos del universo escolar.

Las líneas que siguen pretenden contribuir a estas investigaciones sobre las prácticas de escritura extraescolar, pero esta vez en los jóvenes alumnos de tercer ciclo de la escuela primaria (en edades entre los 7 y los 11 años), mostrando cómo logran construir conjuntamente procedimientos cognitivos ajustados a saberes lingüísticos más amplios.

\section{Los escritos extraescolares de alumnos de primaria}

Los textos de base para este estudio fueron todos recogidos en una misma aula ${ }^{4}$ llama-

\footnotetext{
4 Nunca sabremos cómo agradecer a los profesores de es-
} tas aulas, Catherine y Gérard Rault. Sin ellos, sus alum- da "única" del tercer ciclo, la cual reagrupaba a 28 alumnos pertenecientes a los tres niveles del ciclo: 6 alumnos de CE2, 16 de CMI y 6 alumnos de CM2 (11 niñas y 17 niños). La escuela, ubicada en un departamento semirrural, acoge a niños provenientes de familias contrastadas desde el punto de vista de las categorías socioprofesionales pero homogéneas en el plano lingüístico: todos francófonos, en su mayoría empleados (secretarias), de mandos medios y superiores (entre los cuales había varios profesores), algunos agricultores (granjeros y trabajadores).

Al comienzo, el proyecto de investigación contemplaba la descripción de diversas representaciones del trabajo de escritura, con el fin de determinar cuáles eran las concepciones principales, subyacentes a toda producción escrita, ya se tratara de ellos mismos o de escritores expertos (padres, profesionales...), en la escuela o fuera de ella. Fue a partir de entrevistas realizadas con cada uno de los 28 alumnos como pusimos en evidencia la existencia de prácticas, a veces intensivas, de escritura extraescolar. En efecto, fueron numerosos los alumnos (15 de 28 , es decir el 53,6\% de la clase $^{5}$ ) que nos afirmaron que escribían "cosas", a menudo calificadas por ellos como banales o " sin importancia": escritos cuyo status no tenía sino una relativa legitimidad; algunos llegaron incluso a decir que "eso no es escritura", "me divierte, es todo", "son juegos", "no es serio", "no es como las tareas o los ejercicios para la escuela..." Sin embargo, entre los 28 alumnos, nueve (ocho niñas y un niño) reconocieron que era una práctica importante tanto por la cantidad como por la diversidad de los géneros identificados y por el interés que les suscitó.

nos no habrían estado tan implicados en sus actividades de escritura. Aunque se sorprendieron por la extensión de los textos recogidos y el interés manifestado por sus alumnos por los escritos extraescolares, contribuyeron ampliamente a la constitución de este corpus.

5 En efecto, once niños y dos niñas nos manifestaron que nunca habían producido tales escritos, o que no los habían conservado.

6 Estas afirmaciones sobre la escuela no significan que los maestros de las clases en cuestión descartan cualquier producción escrita, al contrario, en realidad los alumnos no perciben el carácter escolar, como lo veremos enseguida. 
El corpus constituido contiene 118 textos (alrededor de 701 páginas) bastante diversificados (19 géneros diferentes): desde cuentos hasta enunciados de problemas matemáticos; pasando por diarios íntimos, diarios de viaje, poemas, canciones, adivinanzas, e incluso una obra de teatro (cuadro 1 ).

\begin{tabular}{|c|c|c|c|}
\hline n. ${ }^{\circ}$ orden & Género textual & $\begin{array}{l}\mathrm{n} \cdot{ }^{\circ} \mathrm{de} \\
\text { textos }\end{array}$ & $\begin{array}{c}\text { n.o de } \\
\text { páginas }\end{array}$ \\
\hline 1 & chiste & 2 & 2 \\
\hline 2 & diario de viaje & 9 & 178 \\
\hline 3 & canción & 8 & 8 \\
\hline 4 & reseña de lectura & 1 & 2 \\
\hline 5 & cuento & 20 & 59 \\
\hline 6 & adivinanza & 1 & 1 \\
\hline 7 & informe & 1 & 2 \\
\hline 8 & $\begin{array}{l}\text { enunciado de } \\
\text { problema }\end{array}$ & 2 & 3 \\
\hline 9 & $\begin{array}{l}\text { ficción primera } \\
\text { persona }\end{array}$ & 4 & 15 \\
\hline 10 & ficción tercera persona & 2 & 7 \\
\hline 11 & juegos & 18 & 18 \\
\hline 12 & diario íntimo & 1 & 7 \\
\hline 13 & leyenda de foto & 5 & 5 \\
\hline 14 & obra de teatro & 1 & 25 \\
\hline 15 & poema & 21 & 20 \\
\hline 16 & publicidad & 4 & 8 \\
\hline 17 & receta de cocina & 1 & 2 \\
\hline 18 & relato de vida & 16 & 38 \\
\hline 19 & reportaje deportivo & 1 & 1 \\
\hline TOTAL & 19 & 118 & 401 \\
\hline
\end{tabular}

Cuadro 1. Variedad y número de géneros textuales referidos al número de textos y de páginas.

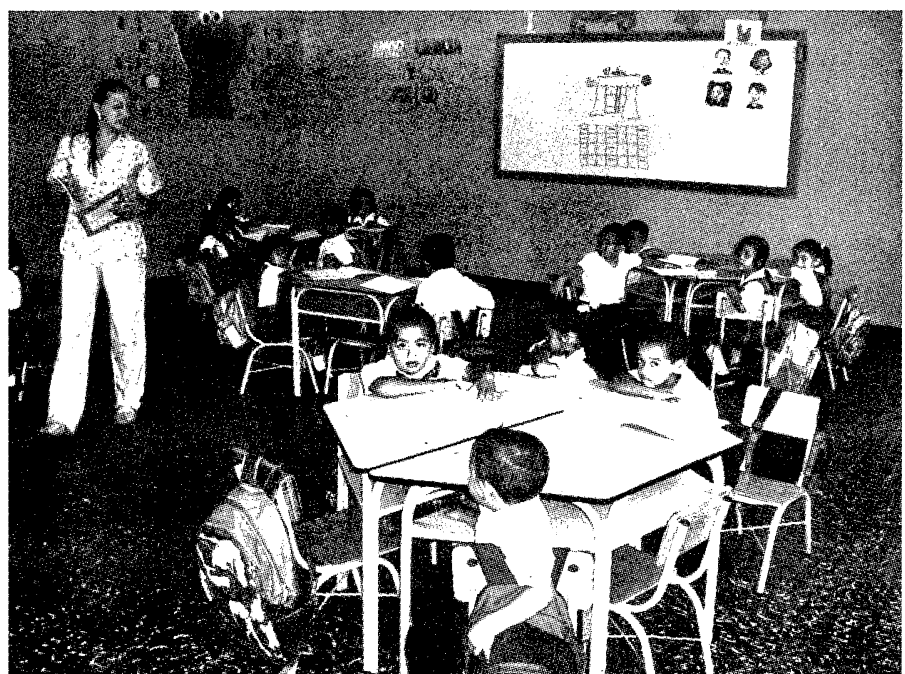

\section{1. ¿Qué estatus darle a estos escritos?}

Los alumnos nos prestaron todos los textos sin ninguna reticencia. ${ }^{7}$ Sin embargo, cinco alumnos (cinco niñas) nos confesaron que no se lo habían dado a leer a nadie, revelando así el carácter secreto, íntimo, impublicable de sus escritos. Los otros, que concedieron una mínima legitimidad a sus producciones, no se sintieron para nada incómodos al entregárnoslos, incluso nos los ofrecieron. ${ }^{8}$

Hay que distinguir dos tipos de comportamientos, pues si bien algunos alumnos (las cinco niñas ya mencionadas) se preocupan por garantizar la intimidad absoluta de sus escritos, los otros tampoco se los dieron a leer a sus padres, a sus hermanos o hermanas o a sus maestros; la razón invocada es que antes que nada sus textos no tienen suficiente interés o crédito para ser entregados a los adultos ("yo no se los doy a la maestra", "ni siquiera mi hermana mayor los lee", "son cosas para mis amiguitas, a mis papás no les interesaría...”). Estas justificaciones con frecuencia están acompañadas de comentarios sobre probables imperfecciones que supuestamente conllevan críticas de parte de estos mismos adultos (porque "no está del todo bien escrito", "no le puse atención a la ortografía, seguro hay errores", "no las volví a escribir [las historias]"). Sin embargo, estos textos a veces pueden circular entre compañeros y compañeras y ser objeto de una correspondencia directa, para prestar o pedir prestado en el azar de los encuentros en la escuela o fuera de ella.

\section{2. ¿Cómo clasificarlos?}

Los soportes de estos escritos son igualmente diversos: a) 18 cuadernos de formato di-

\footnotetext{
7 Solamente una alumna de CE2 no nos dejó su diario y motivó su negativa por escrito: "No tengo deseos de enviarte mi diario porque allí escribí muchas cosas sobre mí. Y además mi mamá no está de acuerdo".

8 Es importante señalar que habíamos invocado el interés de estos textos para "la ciencia" y garantizamos su anonimato. De hecho, ninguno de los nombres citados en los ejemplos que siguen corresponde a los de los niños. $\mathrm{Y}$ evidentemente, los textos les fueron devueltos a sus autores después de haber sido reproducidos.
} 
verso, de preferencia con líneas o cuadrículas; b) 6 conjuntos de hojas blancas, de líneas o cuadros, a veces arrancadas a los cuadernos, pero siempre reunidas; c) 2 libretas más o menos llenàs y completas; d) 2 libretas de notas, y e) un solo archivador (ver detalles en el cuadro 2 ).

Los nueve alumnos más comprometidos en las actividades de escritura privilegian cuadernos bonitos, a veces bastante costosos, mostrando de esta manera que distinguen las actividades escriturales de la clase de las actividades de tiempo libre. Una alumna entre las nueve, posee un diario íntimo impreso comercialmente, con candado y llave para garantizar la intimidad, el cual le fue regalado por sus padres. También allí parece establecerse una distinción entre aquellos escritos que manifiestan un interés preciso y serio por la reescritura, y los otros que no conllevan la misma importancia constante y regular, o que privilegian la rápida ejecución, preocupándose menos de la forma que del soporte de sus escritos.

\begin{tabular}{|c|l|c|c|c|}
\hline $\begin{array}{c}\text { n.o } \\
\text { orden }\end{array}$ & $\begin{array}{c}\text { Soportes de } \\
\text { los escritos }\end{array}$ & Número & $\begin{array}{c}\mathbf{n .}^{\mathbf{o}} \text { de } \\
\text { textos }\end{array}$ & $\begin{array}{c}\mathbf{n .}^{\circ} \text { de } \\
\text { páginas }\end{array}$ \\
\hline 1 & Cuadernos & 18 & 46 & 275 \\
\hline 2 & hojas unidas & 6 & 44 & 82 \\
\hline 3 & libretas & 2 & 8 & 19 \\
\hline 4 & bloc de notas & 2 & 16 & 19 \\
\hline 5 & legajador & 1 & 4 & 6 \\
\hline тотAL & 5 & 29 & 118 & 401 \\
\hline
\end{tabular}

Cuadro 2. Diversidad y número de soportes referidos al número de textos y de páginas.

Los útiles para escribir son igualmente objeto de una atención particular, pero sólo para algunas alumnas: estilógrafos de tinta de color original, azul pálido, turquesa, sepia... una de ellas llegó incluso a utilizar un bolígrafo de varias minas, que acababan de regalarle, para componer una obra de teatro en la cual cada uno de los personajes (más el narrador) se identificó con un color preciso. Los otros alumnos, la mayoría, utilizan los útiles ordinarios (esferográfico) usualmente previstos para el trabajo en clase o en la casa.

En una primera aproximación, nos sorprendimos a la vez por la cantidad de estos escritos producidos fuera de la escuela y por la diversidad de géneros textuales. Dada la importancia cuantitativa de los textos, es necesario realizar varias observaciones. Primero que todo constatamos que las cinco alumnas más prolíficas (en negrilla en nuestro cuadro 3) suman 74 textos sólo entre ellas, es decir, el 63\% del conjunto; traducido en número de páginas, sus textos totalizan 239 , es decir, cerca del $60 \%$.

\begin{tabular}{|c|c|c|c|}
\hline Seudónimo & $\begin{array}{c}\text { Nivel de } \\
\text { clase }\end{array}$ & $\mathbf{n .}^{\circ}$ de textos & n. ${ }^{\circ}$ de páginas \\
\hline Ama & CE2 & 18 & 30 \\
\hline Amo & CE2 & 8 & 19 \\
\hline Cot & CE2 & 16 & 33 \\
\hline Mit & CE2 & 2 & 5 \\
\hline Sol & CE2 & 2 & 28 \\
\hline Clé & CM1 & 11 & 87 \\
\hline Do1 & CM1 & 12 & 30 \\
\hline Hol & CMI & 7 & 48 \\
\hline Jer & CM1 & 8 & 27 \\
\hline Két & CM1 & 17 & 59 \\
\hline Oré & CMI & 4 & 9 \\
\hline Col & CM2 & 5 & 11 \\
\hline Jen & CM2 & 1 & 2 \\
\hline Map & CM2 & 3 & 7 \\
\hline Sib & CM2 & 4 & 6 \\
\hline
\end{tabular}

Cuadro 3. Distribución del número de textos y de páginas producidos por los alumnos.

En su conjunto, los textos se reparten más o menos igual de junio a julio; los períodos de vacaciones escolares no fueron los más propicios para estas producciones intensas. En cambio uno de los factores determinantes para el incremento de estos escritos fuera de la escuela fue el viaje efectuado por el conjunto de los alumnos (salida pedagógica) a Charente-Maritime. Con motivo de este desplazamiento, muchos de ellos redactaron cartas, tarjetas, consignaron notas que no siempre conservaron, pero sobre todo compusieron "libritos de viaje", especies de diarios de viaje, más o menos regulares y con ilustraciones (dibujos, postales, fotos recortadas...). Estos diarios - la mayoría de las veces en forma de cuaderno escolar- son enriquecidos con comentarios, 
anotaciones, ilustraciones, y con frecuencia fueron leídos por otros alumnos, en la medida, por supuesto, que estos alumnos entraron en esta dinámica de intercambio.

En relación con los géneros identificados (cuadro 1), observamos, de hecho, una gran diversidad. Esta diversidad se pone de manifiesto tanto en la selección de diversos soportes: un cuaderno para la novela, otro para los cuentos, un tercero para los poemas, como en la agrupación en un solo soporte: chistes, adivinanzas, poesías, canciones, escritos en un mismo cuaderno o en hojas grapadas.

a) Los relatos de experiencias personales predominan (26 diarios de viaje, diarios íntimos y relatos de vida repartidos en 223 páginas $^{9}$, es decir el 55,6\% del total), pero hemos visto que la proporción de diarios de viaje (178 páginas) explica por sí sola la importancia de esta categoría sobre el conjunto de textos.

b) Los géneros de ficción (cuentos, ficciones de primera persona o ficciones de relato de vida, relatos en tercera persona, obra de teatro) son igualmente importantes y, aunque no son sino 27 textos sobre 118, totalizan más de la cuarta parte de todas las páginas recogidas $(26,4 \%)$.

c) Se encontraron 29 textos poéticos (poemas, canciones) para un total de 28 páginas (es decir prácticamente una página por texto $\mathrm{y}$, por lo tanto, $7 \%$ del conjunto), acompañados con frecuencia de dibujos y de estampas.

d) Los textos humorísticos y/o lúdicos ${ }^{10}(24$ chistes, adivinanzas, enunciados de problemas, juegos, recetas) son igualmente presentados en la misma cantidad de páginas $(26$, lo cual representa $6.4 \%$ del conjunto).

9 Es necesario relativizar el número de páginas y el número de textos porque a veces éstos se reducen a pocas palabras como en las leyendas de fotos, o pueden comprender varias centenas, como en los relatos de vida.

${ }^{10}$ Esta categoría puede parecer heterogénea; en efecto, contrariamente a las otras, ésta corresponde a producciones homogéneas en los documentos recogidos, ya se trate de una libreta, de un cuaderno o incluso de un conjunto de hojas grapadas. Es más, estos relatos están generalmente calificados como tales en los comentarios de los alumnos. e) Finalmente, una serie de textos que podríamos caracterizar por su función de información, pero cuyos elementos, bastante diversos en su forma, son con frecuencia singulares (12 reseñas, una nota enciclopédica, leyendas de fotos, publicidad, reportajes periodísticos -en 18 páginas, es decir el 4\% del conjunto).

\section{De hecho, cada uno de los escritos recogidos muestra que no se trata de una simple reproducción, siquiera parcial, del o de los textos fuente. Los alumnos de nuestro estudio parecen concebir la escritura esencialmente como una actividad creativa.}

\section{3. ¿Cuáles especificidades?}

Más allá de los reagrupamientos efectuados que para algunos podrían parecer muy arbitrarios o poco fundamentados, debemos notar una gran disparidad entre los textos inventados o creados y aquellos que se apoyan sobre referencias ya inscritas, o incluso que parecen simple copia. En sus trabajos sobre los escritos extraescolares de jóvenes, Penloup et al. (1999) constatan que esta segunda categoría, las copias, constituye una parte importante de su corpus (15\% de los alumnos interrogados manifiestan practicar la copia). En relación con nuestro estudio la distancia entre los primeros y los segundos es considerable. Nuestros alumnos se ubican sobre todo en una lógica de creación, ya se trate de relatos de experiencias personales, relatos de ficción, poemas y textos humorísticos o lúdicos. Cuando ellos se refieren a escritos ya presentes lo hacen para aumentarlos, como Alá (ver texto 3, más abajo) apasionado por los animales en general y por las especies amenazadas en particular. Este texto, como muchos otros, heterogéneo desde el punto de vista genérico, revela un traba- 
jo de recreación muy específico, pues se trata de una adaptación -y por lo tanto de una reescriturade datos con carácter enciclopédico. Estos textos, que se apoyan directamente en referentes accesibles, revelan un trabajo de reescritura importante. Son textos con una tendencia informativa: la reseña, el reportaje periodístico o la receta de cocina que mezclan géneros. La receta de cocina escrita por Két (texto 2 más abajo) es reveladora de esta mezcla pues se apoya en una experiencia precisa: la elaboración de una torta de nueces hecha por la alumna con tres de sus amigas. En algunos otros casos, el texto es recuperado de memoria, como en el chiste anotado por Amo (texto 1), en donde se puede considerar que, incluso si la fuente es un texto oral escuchado, su transcripción revela, ella misma, una escritura original.

De hecho, cada uno de los escritos recogidos muestra que no se trata de una simple reproducción, siquiera parcial, del o de los textos fuente. Los alumnos de nuestro estudio parecen concebir la escritura esencialmente como una actividad creativa. Por supuesto que los interrogamos sobre este aspecto y las entrevistas confirman sus escritos. En cada entrevista les preguntamos sobre aquello que habían copiado o consignado en sus libretas, cuadernos, hojas grapadas y aquello que no era de su propia autoría. Sólo un alumno nos mostró un trabajo de copia aunque limitado a un solo poema amoroso, escrito en un cuaderno sin mayores consecuencias.

¿Cómo podemos explicar una distancia tal entre nuestro corpus y el recogido por otros investigadores en el nivel secundario?

En primer lugar, parece que contamos con una población de niños relativamente homogénea desde el punto de vista sociológico. De hecho, se parecen a aquellos alumnos de escuela primaria o de secundaria descritos en investigaciones etnolingüísticas adelantadas por otros (Barré de Miniac, Cros y Ruiz, 1993, y Lahire, 1995), de quienes no se diferencian mucho en sus comportamientos frente a la escritura; la pertenencia social y cultural de sus familias parece, desde este punto de vista, bastante homogénea. Nuestros alumnos se acercan sensiblemente a aquellos sujetos que viven la escritura como algo poco tensionante, sobre todo en el plano formal. De hecho, los padres y, sobre todo, los dos profe- sores de su clase, conciben los aprendizajes de la producción escrita de manera abierta, brindando mucha atención a los efectos producidos sobre los lectores, a la pertinencia enunciativa y al ajuste de los escritos a las consignas colectivas o a los proyectos individuales, sin que esto jamás frene la escritura. Aunque esto no quiere decir evidentemente que no presten ninguna atención a esta actividad, como lo veremos enseguida.

Parece entonces que a un "estilo" de.aprendizaje corresponde un "estilo" de alumnos. Estos dos estilos igualmente abiertos amplifican el fenómeno de continuidad observado entre las prácticas de escritura escolares y no escolares. De hecho, los segundos, los textos producidos fuera de actividades de clase e identificados así por los alumnos, con frecuencia están inspirados en los primeros, los textos escolares. ${ }^{11}$ Es frecuente que un trabajo particular en la escuela sobre una forma poética (por ejemplo el haiku, $y$ también un proyecto o un tema (por ejemplo la prensa, el hambre en el mundo, las especies de animales en vías de extinción) conlleven en los alumnos un interés por la producción de textos fuera del aula, del mismo género o del mismo tema. Así, en ciertos períodos del año, los alumnos -sobre todo los más jóvenes de CE2 y de CMI- compusieron poemas cortos, luego páginas de publicidad o afiches de actualidad y, más tarde, una nota enciclopédica.

Para describir precisamente estos procesos nos pareció necesario establecer una metodología pertinente, pues los datos estadísticos y nuestras tipologías no dicen gran cosa si no las podemos complementar con análisis cualitativos que enfrenten más directamente los problemas de escritura registrados, las soluciones encontradas y los procedimientos utilizados o accesibles.

\section{Por una metodología adaptada}

Desde la recolección de los primeros textos, nuestra metodología ha evolucionado hacia un procedimiento que podríamos calificar

\footnotetext{
${ }^{11}$ No podemos entrar en el detalle de las prácticas de producción escrita desplegadas en esta clase. Sin embargo, las describimos en otro escrito (Bore y David, 1996) al que los lectores pueden acceder.
} 
de "naturalista" en el sentido en que se pone a los alumnos en situación de comentar sus propias producciones. ${ }^{12}$ Los interrogamos sobre sus escritos no escolares ${ }^{13}$ en el curso de las entrevistas semidirectivas realizadas por nosotros y registradas en audio ${ }^{14}$, no más de diez días después de la escritura de los textos. De hecho, esta actividad no perturba mucho los dispositivos didácticos ni la organización pedagógica de la clase.

De esta manera, constituimos un doble corpus que pone frente a frente los textos producidos por los alumnos y las autoexplicaciones que los acompañan o los amplían. Esencialmente, es sobre la base de estas autoexplicaciones o "explicaciones metagráficas" (en adelante EM) que interpretamos la mayor parte de las decisiones tomadas por los niños. Efectivamente, las EM transcritas y categorizadas fueron analizadas en términos de procedimientos o estrategias en función de un problema lingüístico. Para facilitar el tratamiento de estos datos, escogimos una unidad: la secuencia (se en nuestro corpus), que asocia a cada problema lingüístico (por ejemplo: la homofonía verbal, la anáfora pronominal, las concordancias tempo-verbales) uno o varios procedimientos de resolución (por ejemplo: la revisión implícita vs. explicita, la presencia vs. la ausencia de modificación, también el tipo y la dimensión de esta modificación, por ejemplo: el reemplazo de una palabra o de un morfema, la adición de un sintagma o de una frase...). Cada secuencia se presenta así bajo la forma de una EM, o de un encadenamiento de EM limitado a la resolución del problema y a los procedimientos identificados.

Como vemos, esta metodología surge en primer lugar de un tratamiento cualitativo de los datos, aunque posteriormente permite un im-

${ }^{12}$ Nos inspiramos en protocolos ya utilizados en investigaciones precedentes y en curso (Jaffré y Ducard, 1996; David, 1997; David y Jaffré, 1997, y David, 2001).

${ }^{13}$ No limitamos nuestro estudio solamente a estos escritos, a pesar de que este proyecto se centra esencialmente sobre este tipo de producciones. Es más, desearíamos poder comparar estos textos originales con aquellos elaborados en el marco de los aprendizajes de la clase.

${ }^{14}$ Se realizaron algunos videos, más por necesidades de formación que por necesidades de este análisis. portante trabajo estadístico sobre cientos de secuencias inventariadas. De hecho, las entrevistas se realizaron a partir de uno o varios problemas identificados en los textos $-y$ a veces incluso en ausencia de ellos, como cuando les preguntamos igualmente sobre las formas exitosas-, por lo tanto no podemos arriesgarnos a concluir sobre la realidad del desarrollo de los procesos realizados antes de describirlos exhaustivamente. La acumulación de secuencias de un mismo tipo y por un periodo preciso permitirá elaborar un modelo genético de la reescritura que no desconoce ni el nivel de las construcciones cognitivas ni el nivel de los funcionamientos lingüísticos.

Es más, si hemos preferido poner a prueba esta metodología es para acceder más directamente a los funcionamientos cognitivos evitando interpretaciones, incluso extrapolaciones a partir de las marcas observadas en la superficie de los textos, como es el caso frecuente en la mayor parte de estudios sobre estos mismos componentes. ${ }^{15}$

Precisemos de todos modos que si esta metodología ofrece el interés de mantener a los alumnos en su ambiente escolar, con el fin de evaluar precisamente los procedimientos de adquisición efectivos o potenciales, no descuidamos el hecho de que nuestras intervenciones, a través de las diferentes entrevistas realizadas, pueden incidir sobre las adquisiciones en curso. Los alumnos en efecto fueron invitados a comentar, a explicar e incluso a argumentar sus soluciones gráficas. De esta manera, fueron llevados a revisar sus escritos, lo que aumentó la precisión de sus actos de lectura y escritura y más globalmente sus capacidades metalingüísticas. Se da por descontado que estos efectos se tuvieron en cuenta en el análisis de los datos e igualmente están en la base de nuestras proposiciones didácticas en este campo.

\footnotetext{
15 Para una síntesis y una discusión de las diferentes metodologías utilizadas para el estudio de la adquisición de la escritura, ver el volumen Estudio de lingüistica aplicada (David y Fayol (edits.), 1996) y nuestro capítulo en Fabre (dir.), 2000.
} 


\section{Escritura y reescritura extraescolares}

$¿$ Existen procedimientos de escritura y de revisión específicos para los escritos producidos fuera del marco escolar? Por supuesto que nos hemos interrogado sobre la pertinencia de esta pregunta ya que podría ser evidente que un escrito personal e incluso íntimo, al menos que no sufra la presión de la lectura de un adulto sino la de un par, no presente el mismo grado de perfección que un escrito entregado en clase a su maestro. La evidencia mostraría, en efecto, que un alumno entre 8 y 11 años, que tiene una clara conciencia de las exigencias relacionadas con la escritura de su lengua, que posee ya un cierto número de concepciones más o menos ajustadas a la norma ortográfica y que al menos ha asumido ya la necesidad de una enunciación legible o comunicable a otros, presta una atención menos cuidadosa a la elaboración de estos textos "íntimos" o "privados" que a aquellos textos elaborados en la esfera escolar. Para decirlo de una vez, nosotros nos esperábamos este tipo de efectos, sin embargo, veremos que ese no fue el caso.

\subsection{De la pertinencia de los procedimientos ortográficos}

Todos los textos recogidos para nuestro estudio tienen una legibilidad sorprendente. No existe uno solo que no hayamos podido decodificar. Ciertamente los problemas de escritura son numerosos y las modificaciones son consecuentes, pero ninguno presenta problemas ortográficos que oscurezcan su comprensión. Todos tienen las mismas características, muestran las mismas lógicas subyacentes, proponen soluciones gráficas ni más ni menos normalizadas que aquellas de sus producciones escolares -trátese de estos alumnos como de otros de su misma edad-. ${ }^{16}$ El texto de Amo, en esta perspectiva, es ejemplar. Se trata de un chiste escuchado y reproducido después de un intercambio con una amiga.

\footnotetext{
${ }^{16}$ Que ya han sido objeto de descripciones más o menos completas (para una revisión de estos trabajos ver Jaffré, 1992).
}

\author{
Texto n. ${ }^{\circ}$ 1. Fragmento de libreta "Chiste". Amo \\ (CE2: 9 años 1 mes) \\ Une idanme amemene «sa ch'mene chez un \\ horloger mesieur ma chinne est maki) \\ [malade] \\ «mais madame eeje suis hoologer et pas vé- \\ térinère» \\ «Ustement ma chinne sarrête toute les sis \\ minute»
}

Traducción aproximada y seminormalizada:

Una señora lleva "su perra a un relojero señor mi perra está mala)

\section{[enferma]}

"pero señora eeh... yo soy relojero y no veterinario"

minutos"

"justamente mi perra se para cada seis

En este texto no falta ningún elemento. Las oraciones se comprenden, el conjunto muestra una organización coherente. La entrevista que sigue permitió aclarar los procedimientos ortográficos implementados, especialmente a través de enmendaduras. ${ }^{17}$

sQ 1: Amo (CE2: $9 ; 1$ ans)

Extraits observés. «...sa ch'mene... ma chinne... ma chinne..."

Amo: là (montre sa chmene) je me suis trompée je croyais qu'il fallait deux $n$ mais il fallait un e pour faire [jen] c'est comme pour une - au féminin ça fait pas [je].

Amo: là (montre la deuxième occurrence : ma cbinne) j'ai oublié - il faudrait mettre un

\footnotetext{
${ }^{17}$ Debido a la particularidad del sistema ortográfico y gramatical de la lengua francesa, no es posible realizar una traducción de todos los textos de los ejemplos, ni de todas las secuencias de entrevistas. Hicimos la traducción completa del primer ejemplo y sus secuencias EM. Del segundo apartado transcribimos el texto original con su traducción y solamente una traducción parcial del análisis que permiten una comprensión global de las explicaciones metagráficas allí presentes. El apartado 4.3 del texto original lo omitimos por considerar que las reflexiones metalingüísticas son específicas del francés y por lo tanto de muy difícil traducción al español.
} 
E comme au début - et là aussi (montre la troisième : ma chinne).

Fragmentos observados: “...sa ch'mene... ma chinne... ma chinne..."

Amo: allá (muestra sa chmene) me equivoqué: yo creía que se necesitaban dos $n$ pero lo que faltaba era una e para hacer [jen] es como une -en femenino no va [je]

Amo: allá (muestra el segundo caso: ma chinne) se me olvidó -faltaba poner una $\mathrm{E}$ como al comienzo- y allá también (muestra el tercer caso: ma chinne).

$\mathrm{E}$ l intercambio revela igualmente que un mismo procedimiento se extiende a otras categorías gramaticales, incluso si la explicación sigue una lógica semántica: la e prototípica del femenino, en francés, no señala directamente la regla de concordancia del género correspondiente, como se ve en la siguiente secuencia.

SQ 2: Amo (CE2 9; 1 ans)

Extraits observés : «... maiaé [malade]»

Amo: malade c'est au féminin aussi il faut un E c'est la chienne qui est malade autrement on croit que c'est le-le vétérinaire qui est malade.

Fragmentos observados: “... maiaé [malade]", enferma.

Amo: malade es en femenino, también se necesita una E: es la perra la que está enfer$\mathrm{ma}$, si no uno piensa que es el veterinario el que está enfermo.

Estas EM son del mismo orden que aquellas que estudiamos en los textos producidos en las actividades de clase por alumnos de la misma edad, tanto en aprendizajes específicos de producción escrita como en el uso de textos en otras áreas (Jaffré y David, 1999). Como puede verse, los procedimientos identificados son construidos progresivamente en el sistema. Éstos revelan la aplicación de lógicas cognitivas a veces titubeantes, pero siempre ajustadas a los problemas lingüísticos encontrados.

\subsection{Densidad de las operaciones y procedimientos de reescritura}

Nos sorprendió la gran cantidad de tachones en la mayor parte de los textos. Como lo habíamos dicho anteriormente, los alumnos fueron sensibilizados al trabajo de revisión debido a que las mismas entrevistas movilizan una actividad metalingüística sostenida, además de que las huellas de esta actividad no son menos importantes. Así, en el texto de Két las enmendaduras denotan relecturas en varias fases de la transcripción del texto y con grados de intervención más o menos profundos y globales.

\section{Texto n. 2 2. Fragmento del legajador "Las recetas" (hojas grapadas). Két (cm1: 8 años 11 meses)}

CM2 : GấTEAU AU[X] NOISETTES

la un yaourth nature

$2 p o t[s]$ de farine

3ceufs

2 pot $[s]$ de sucre 1

pot de noisette pilés 1

sachet de levure 1/2

pot d'buile

faire cuire au four 30 minutes 7

thermostat $[s]$

recette de : marie-pierre - bélène Audrey

tout[s] le monde c'est régaler[és] quand nous les avons manger [és] l'après-midi.

CM2 TORTA DE NUECES

Un yogurt natural

2 tazas de harina

3 huevos

2 tazas de azúcar 1

taza de nuez machacadas 1

sobre de levadura $1 / 2$

taza de aceite

cocinar al horno 30 minutos 7

termostato[s]

receta de: marie-pierre/hélène Audrey

todo el mundo se saboreó

cuando las comimos en la tarde. 


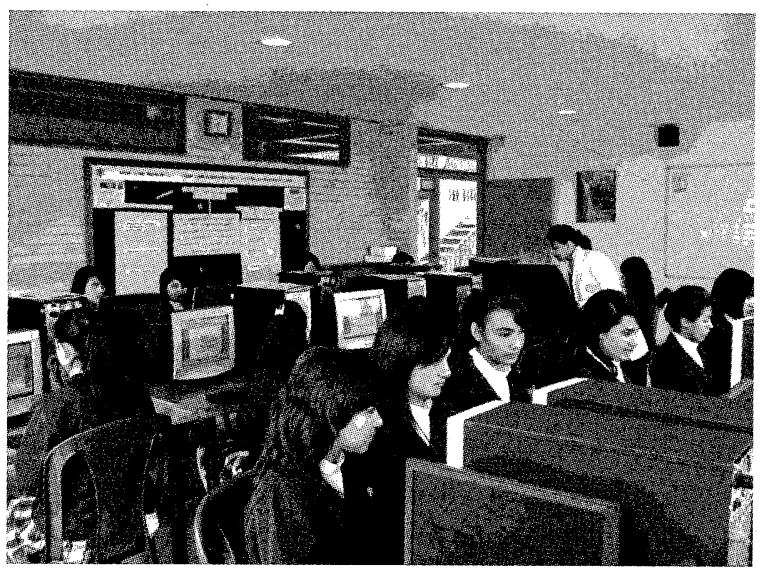

La mayor parte de las enmendaduras ${ }^{18}$ corres- $^{-}$ ponden a relecturas posteriores a la transcripción del texto (de ahora en adelante designadas como variantes de lectura $)^{19}$, cuatro adiciones: $A U[X] \ldots$ $\operatorname{pot}[s] \ldots$ pot $[s] \ldots$ thermosta $[s]$, y tres substituciones: tout $[s]$... régaler $[e ́ s]$.. manger $\left[e_{s}\right]$...; solamente una substitución relacionada con el cambio de determinante: $l a-u n \ldots$.., se realizó en el momento de la escritura (i.e. variante de escritura también según la distinción tomada de Grésillon, 1994).

Igualmente observamos que estas variantes de lectura manifiestan ajustes importantes esencialmente en relación con la marca de número. Sin embargo, un análisis lingüústico más fino muestra que estas modificaciones tocan al tiempo la clase de los nombres, determinantes y verbos hasta la precisión de los participios pasados. De hecho, la entrevista sobre este texto reveló que estas variantes no fueron propuestas al azar, sino que fueron el resultado de cálculos inteligentes, aplicados intencionalmente según una lógica aquí también justificada.

Las variantes de reescritura, los niveles de intervención sobre el texto y los tipos de enmendaduras confirman la precisión del trabajo reflexivo. De ninguna manera se trata de un escrito compuesto en el encuentro azaroso de

\footnotetext{
${ }_{18}$ Para una mejor comprensión, las enmendaduras se señalan entre paréntesis cuadrados [ ] (nota de las traductoras).

19. Según la distinción propuesta por los investigadores del Instituto de Textos y Manuşcritos Modernos (ITEMCNRS) expuesta por Grésillon (1994). Esta distinción ha sido ampliada a los borradores escolares por Fabre (1990) y por nosotros mismos en diferentes estudios (David, 1994) y la presente contribución.
}

ideas y palabras. Si los cálculos ortográficos no se realizaron en el curso mismo de la escritura, sí se hicieron durante la relectura. Las EM confirman y aclaran los recorridos realizados. Ellas muestran que los procedimientos se construyen $y$ funcionan de manera eficaz mientras que otros emergen para combinarse eventualmente con los precedentes. De hecho, los saberes construidos se inscriben en el momento y durante la tarea que los requiere, en este caso la revisión del texto. No pueden ser movilizados todos al mismo tiempo o, por lo menos, no todos en un mismo acto de escritura.

\section{En conclusión}

Nuestro estudio se propuso mostrar que los escritos extraescolares y los escritos escolares no difieren ni en su pertinencia discursiva, ni en su adecuación de ortografía y composición. Para ello utilizamos el análisis conjunto de las variantes de reescritura que fueron identificadas en la superficie de los textos y de las EM que las acompañan, y exploramos los hechos de lengua delimitados con precisión y los procedimientos psicolingüísticos adquiridos o en emergencia en el trabajo de escritura-reescritura. De hecho, para nosotros, el estudio de los textos terminados o en gestación y de los comentarios realizados por los alumnos posee un valor heurístico. Estas EM prueban, si fuera necesario, que estos procedimientos de escritura son accesibles a la conciencia de los sujetos y que resultan de un trabajo cognitivo totalmente descriptible.

Estas mismas observaciones, que no se limitan evidentemente a los fenómenos y ejemplos estudiados aquí, muestran igualmente que los saberes construidos por los aprendices no surgen de la nada, que no son nunca creaciones ex nibilo. Diremos entonces que sus conocimientos lingüísticos y sus capacidades para escribir no son el resultado de descubrimientos aleatorios o impuestos sino más bien resultado del despliegue progresivo y regular de procedimientos descritos en parte en este trabajo. Parece, en efecto, que los alumnos proceden mediante organizaciones y reorganizaciones sucesivas de trayectorias coherentes y ajustadas a los problemas lingüísticos 
encontrados. Así, como ya lo vimos, las soluciones más efectivas pueden provenir de los unos o de los otros. Pero más allá de las soluciones identificadas, es la capacidad para razonar, para jugar con las variaciones del escrito, para ampliar la aplicación de las reglas -o al contrario para restringirlas-, en resumen, para desplegar las actividades metalingüísticas extendidas y coordinadas, lo que demuestra que los alumnos no han sido nunca pasivos en sus adquisiciones.

¿Qué deducir en términos de aprendizaje? ¿Cuáles aplicaciones didácticas formular?

Antes que nada nos parece fundamental tener en cuenta las representaciones construidas por los alumnos a partir de las diferentes tareas de escritura, así como sobre los objetos lingüísticos tratados: las unidades y sus categorizaciones, las regularidades y las variaciones, los funcionamientos sintácticos y las organizaciones textuales... De hecho, si los estudiantes no construyen nuevos conocimientos partiendo de la nada, el maestro debe evaluar entonces las conceptualizaciones ya adquiridas y las que les son accesibles. El estudio combinado de los procedimientos de escritura-reescritura y de las EM nos parece que es un buen medio para lograr esto.

Los textos producidos fuera de situaciones escolares y analizados en este documento muestran que existe una continuidad de los aprendizajes. Las habilidades de redacción no se reducen cuando las obligaciones escolares son menos exigentes. Podemos de esta manera asumir las actividades solidarias yendo de lo escolar hacia lo extraescolar y recíprocamente, y de esta manera invitar a los estudiantes a escribir en todas las situaciones de la vida mostrando que esta actividad no se detiene en las puertas de la escuela.

De esta manera, el estudio de los diferentes géneros textuales ganaría en calidad si los escritos personales pudieran ser evocados, o expuestos, con la misma importancia que las producciones escolares. Las actividades de clasificación o selección de textos serían entonces mejor comprendidas porque se apoyarían sobre un trabajo real de producción y no solamente de recepción. Los estudiantes podrían entonces sostener discursos, elaborar explicaciones y argumentos basados en estas experiencias escriturales personales. Mejor aún, ciertos alum- nos cuya escritura intensiva fuera de la escuela es manifiesta, tendrían así la posibilidad de ver valorados sus escritos y de encontrar allí un cierto reconocimiento institucional. En esta perspectiva, la escritura de invención, reducida con frecuencia a la reproducción más o menos intencional de estereotipos, ganaría en calidad si pudiera renovarse en otras fuentes, especialmente en estas experiencias originales de escritura. Por supuesto que no se trata de escolarizar $-\mathrm{y}$ menos aún de evaluar- escritos que generalmente no tienen sentido sino en sus usos íntimos, personales, o en intercambios secretos con otro, sino de mostrar que tales producciones existen y constituyen en sí mismas géneros (la correspondencia, los diarios íntimos) y prácticas literarias (la reescritura de sus propios textos, por ejemplo) que se desarrollan según las épocas, los autores y el interés de los lectores.

Más allá de esto, nos parece importante invitar a los alumnos a interrogarse sobre sus propias prácticas escriturales, sin limitarlos, tal como lo hacen espontáneamente en las producciones escolares. Este examen personal con frecuencia es decisivo para ciertos alumnos, poco inclinados a reconocer su trabajo, reconocer en el doble sentido de encontrarse con sus escritos o volver sobre ellos y de otorgarles un cierto valor. Para los mismos niños, la verbalización de sus recorridos personales, incluso de sus historias familiares, puede ser suficiente para revisar representaciones fijas y rehabilitar prácticas o géneros "ordinarios", enmascarados por un imaginario cultural construido escolarmente. Se tratará entonces de mostrar que toda producción escrita tiene un sentido y puede dar sentido en función de los usos y contextos de su emisión-recepción, ya se trate de una serie de chistes, de adivinanzas, canciones inventadas o copiadas, así como recopilaciones de cuentos o de poemas, de una novela, de una reseña de lectura... generalmente mejor reconocidos por la escuela.

En cuanto a los aprendizajes, pensamos que esta investigación sobre los escritos extraescolares y su solidaridad con los escritos escolares puede ayudar a los maestros a concebir actividades de producción escrita necesariamente inscritas en un trabajo de reescritura. Para ellos los diferentes textos y borradores recogidos pueden 
servir de base para la construcción de saberes más rigurosos, trátese del descubrimiento de los principios básicos de la escritura de su propia lengua o del acercamiento a funcionamientos discursivos particulares. Éstos podrían entonces conducir a los estudiantes a identificar los fenómenos lingüísticos accesibles y a construir saberes dinámicos que permitan el paso de una operación a la otra. Podría ser el análisis de una palabra para su inserción en una frase o en un texto; podría ser igualmente el tratamiento de un mismo hecho de lengua en las lógicas semánticas, ortográficas y enunciativas. Hemos mostrado que los fenómenos de concordancia ortográfica del francés necesitan de un trabajo cognitivo de esta naturaleza. Finalmente, los estudiantes deberán ser capaces de hacer explícitas las relaciones que tejen los conocimientos intuitivamente descubiertos con los enseñados escolarmente. Será necesario para esto avanzar a tientas al escribir y reescribir, al ajustar progresivamente' sus razonamientos a los diferentes fenómenos lingüísticos inherentes a la composición de sus textos. Para ello es necesario acrecentar sus saberes metalingüísticos, lo que supone un diálogo constante con los otros alumnos y con sus maestros, inscritos todos en una reflexión profunda tanto sobre el funcionamiento de los textos y la génesis de la escritura como sobre el funcionamiento de la escritura y la génesis de los textos. Ó

\section{Bibliografía}

Barré de Miniac, C. ; Cros, F. y Ruiz J. (1993), Les collégiens et l'écriture. Des attentes familiales aux exigences scolaires, París, INRP-ESF.

Besse, J. M. (1995), L'Écrit, l'école et l'illettrisme, París, Magnard.

Blanc, D. (1996), "Le temps des cahiers", en Barré de Miniac, C., Vers une didactique de l'écriture, París, De Boeck-INRP,

Bonnet, C. y Gardes-Tamine, J. (1990), L'Enfant et l'écrit. Récits, poésies, correspondances, journaux intimes, París, Armand Colin.

Bonnet, C. ; Corblin, C. y Élalouf, M. L. (1998), Les Procédés d'écriture chez les élèves de 10 à 13 ans, un stade de développement, VersallesLausana, IUFM de Versailles.
Bore, C. (1998), Choix énonciatifs dans la mise en mots de la fiction. Le cas des brouillons scolaires [tesis de doctorado], Grenoble, Université Stendhal, Doctorado en Ciencias del Lenguaje.

- y David, J. (1996), “Les différentes opérations de réécriture: des brouillons d'écrivains aux brouillons d'élèves", en : Plane, S. y Turco, $\mathrm{G}$. (edits.), De l'évaluation à la réécriture, $\mathrm{Pa}$ rís, Hachette- INRP.

Bousquet, S., et al. (1999), "Acquisition de l'orthographe et mondes cognitifs", en $R e$ vue française de pédagogie, núm. 126, París, INRP.

Brissaud, C.y Sandon,J.M.(1999), “L'acquisition des formes verbales en / $\mathrm{E} /$ à l'école élémentaire et au collège, entre phonographie et morphographie", en Langue française, núm. 124, París, Larousse.

Bucheton, D. (1995), Écriture, réécritures. Récits d'adolescents, Berna-París, Peter Lang.

David, J. (1994), "Écrire, c'est réécrire. De la pertinence des ratures chez l'écolier", en $L e$ Français aujourd'bui, núm. 108, París.

- (1997), "Écriture et acquisition. Étude de procédures graphiques et d'interactions verbales chez des enfants de 6 à 8 ans", en $C a$ hier du français contemporain, núm. 4. Saint Cloud, ens.

- (2001), "Les procédures orthographiques dans les productions écrites des jeunes enfants", en Revue des Sciences de l'éducation [en prensa], Quebec, Université de Québec à Montréal.

- y Farol, M. (edits.), (1996), "Comment étudier l'écriture et son acquisition?" en Études de linguistique appliquée, núm. 101, París, Didier-Érudition.

- y Jaffré, J. R. (1997), "Le rôle de l'autre dans les procédures métagraphiques", en Recherches, núm. 26, Lille.

- y Plane, S. (edits.), (1996), L'Apprentissage de l'écriture de l'école au collège, París, PuF.

Fabre, C. (1990), Les Brouillons d'écoliers ou l'entrée dans l'écriture, Grenoble, CEDITELL'atelier du texte.

-(dir.), (2000), Apprendreà lireles textes d'enfants, Bruselas, De Boeck \& Duculot. 
Farol, M. (1997), Des Idées au texte. Psychologie cognitive de la production verbale, orale et écrite $e_{2}$ París, PuF.

Ferreiro, E. (1988, 2000), "L'écriture avant la lettre", en Sinclair, H. (edit.): La Production de notations chez le jeune enfant, París, PUF, versión revisada en Ferreiro, E. (2000), L'Écriture avant la lettre, París, Hachette.

- y Teberosky, A. (1979, 1982), Los sistemas de escritura en el desarrollo del niño, México, Siglo XXI.

Fijalkow, J. (dir.), (1990), Décrire l'écrire, Toulouse, CRDP-Presses universitaires du Mirail.

Grésillon, A. (1994), Éléments de critique génétique. Lire les manuscrits modernes. París, PuF.

Hay, L. (1979), Essais de critique génétique, París, Flammarion.

- (1992), Didactiques de l'orthographe, París, Hachette-INRP.

- y David, J. (1999), "Le Nombre: essai d'analyse génétique”,en Langue française, núm. 124, París, Larousse.

- y Ducard, D. (1996), "Approches génétiques et productions graphiques", en; Études de linguistique appliquée, núm. 101, París, Didier-Érudition,

Lahire, B. (1995), Tableaux de familles. Heurs et malheurs scolaires en milieux populaires, París, Gallimard-Le Seuil.
Penloup, M. C. (edit.);-(1994), La Rature n'est pas un raté. Plaidoyer pour le brouillon, Ruán, MAFPEN.

- (1999), L'Écriture extrascolaire des collégiens. Des constats aux perspectives didactiques, $\mathrm{Pa}-$ rís, ESF.

- (2000), La Tentation du littéraire. Essai sur le rapport à l'écriture littéraire du «scripteur ordinaire», París, Didier-Érudition.

Piolat, A. y Pélissier, A. (edits.), (1998), La Rédaction de textes. Approche cognitive, Neuchâtel-París, Delachaux et Niestlé.

Plane, S. (1994), Écrire au collège. Didactique et pratiques d'écriture, París, Nathan.

- y Turco, G. (edits.), Groupe eva (1996), De l'évaluation à la réécriture, París, HachetteINRP.

Reuter, Y. (1996), Enseigner et apprendre à écrire, París, EsF.

Simon, J. (1973), La Langue écrite de l'enfant, París, PUF.

Tauveron, C. (1995), Le Personnage. Une clef pour la didactique du récit à l'école élémentaire, Neuchâtel-París, Delachaux et Niestlé.

Zesiger, P. (1995), Écrire: approches cognitive, neuropsychologique et déve-loppementale; París, PuF. 